\title{
Zur Einleitung in die Sammlung
}

\section{„Kölner Studien zum Staats- und Wirtschafisleben““}

Unter diesem Namen wird eine Sammlung veröffentlicht, die von den unterzeichneten Dozenten der Handels-und der Verwaltungshochschule zu Köln bei dem mitunterzeichneten Verlag herausgegeben wird.

Sie hat die Aufgabe, wissenschaftliche Untersuchungen der Herausgeber und besonders auch die Arbeiten der in den Hochschulseminaren tätigen Studierenden der Öffentlichkeit zu übermitteln. Die Herausgeber wurden zu ihrem Vorgehen namentlich dadurch veranlaßt, daß zahlreiche Studierende der beiden Hochschulen Leistungen hervorbringen, die auf besonderer wissenschaftlicher und beruflicher Sachkenntnis beruhen, und die daher geeignet sind, die wissenschaftliche Erkenntnis zu fördern und zu bereichern.

Die „Kölner Studien“ sollen Stoffe aus den für die genannten Hochschulen charakteristischen Wissenschaften enthalten, das sind: Nationalökonomie, Wirtschaftsgeschichte, Rechts- und Versicherungswissenschaften, Genossenschaftslehre und Geographie. Von der Berücksichtigung der Privatwirtschaftslehre ("Handelstechnik"), die ein anderes wesentliches Arbeitsgebiet des akademischen Unterrichts beider Anstalten umfaßt, kann dabei abgesehen werden; denn diese besitzt bereits in der von Prof. Schmalenbach herausgegebenen "Zeitschrift für handelswissenschaftliche Forschung" ein Organ, das namentlich für die Kölner Handelshochschule Aufgaben löst, die zum Teil mit denen der "Studien" parallel laufen. 
Die „Kölner Studien" sollen in zwangloser Folge in einzelnen Heften erscheinen, die in der Regel eine Einzeldarstellung enthalten. Gelegentlich ist jedoch auch die Zusammenfassung mehrerer kleiner Arbeiten in einem Heft vorgesehen.

P. ABERER. CHR. ECKERT.

J. FLECHTHEIM. JUL. FRIEDRICH. ED. GAMMERSBACH. H. GEFFCKEN. K. HASSERT. J. HIRSCH. B. KUSKE.

PAUL MOLDENHAUER. F. STIER-SOMLO. ADOLF WEBER. K. WIEDENFELD.

A. WIERUSZOWSKI. W. WYGODZINSKI.

A. MARCUS UND E. WEBERS VERLAG (Dr. jur. ALBERT AHN.) 